\title{
Pneupard: A Biomimetic Musculoskeletal Approach for a Feline-inspired Quadruped Robot
}

\author{
Andre Rosendo, Shogo Nakatsu, Kenichi Narioka and Koh Hosoda
}

\begin{abstract}
Feline locomotion combines great acrobatic proficiency, unparalleled balance and higher accelerations than other animals. Capable of accelerating from 0 to $100 \mathrm{~km} \mathrm{~h}^{-1}$ in three seconds, the cheetah (Acinonyx jubatus) is still a mystery which intrigues scientists. Aiming for a better understanding of the source of such higher speeds, we develop a biomimetic platform, where musculoskeletal parameters (range of motion and moment arms) from the biological system can be evaluated with air muscles within a lightweight robotic structure. We performed experiments validating the muscular structure during a treadmill walk, successfully reproducing animal locomotion while adopting an EMG based control method.
\end{abstract}

\section{INTRODUCTION}

Animal locomotion is continually evolving, for the sake of survival, creating faster and more versatile forms. On the Felidae family, which have always been a remarkable example of optimized locomotion, we could mention the leopard (Panthera pardus), which, although not as strong as the lion (Panthera leo), uses its great climbing skill to pull its prey to the top of a tree, away from stronger predators. Felids, similarly to dogs, horses and humans, rely on their morphology, which defines how good they will be performing tasks.

Apparently, this degree of specialization of an animal is associated with its mass, dimensions and, more importantly, muscular structure. In [1], a comparison among members of the Felidae family is drawn, showing locomotion and morphologic similarities with felines ranging from 4 to 200 $\mathrm{kg}$. In [2] and [3] Wilson shows a morphologic comparison between cheetahs and greyhounds, both with similar dimensions and weight, but with different muscular structure and, consequently, different maximum speed.

Research concerning muscular role on feline locomotion has been progressively evolving in the last 50 years, with great contributions from Engberg and Lundberg [4], Goslow [5], English [6] and Herzog [7], approaching the problem through observation and measurement of stepping cats. Although a high degree of knowledge on muscle roles is obtained, the highly redundant morphology of muscular systems compels us to believe that phenomena could be better understood if artificially reproduced, leading to a constructivist approach.

Drawing inspiration from nature has been one of the major forms of human creation and, likewise, roboticists. Among many existing quadruped robots (e.g., [8] [9]) we

This work was partially supported by KAKENHI Kiban(S) 23220004

The authors are with Multimedia Department, Faculty of Information Science and Technology, Osaka University, 1-5 Yamadaoka, Suita, Osaka, Japan. andre.rosendo@ist.osaka-u.ac.jp can categorize many different degrees of biomimicry. Robots such as [10] and [11] allow the study of a cheetah by simply reproducing the same movements, not accounting for muscular contribution, having a low degree of biomimicry. Approaches such as [12] and [13] were more thorough, adopting monoarticular muscles as actuation means, while [14] adopted exclusively biarticular muscles for this purpose. Biologists theorize that biarticular muscles hold an essential role on musculoskeletal systems, transfering loads between joints [15]. This way a higher degree of biomimicry would be possible with redundant structures, combining mono and biarticular muscles.

The most biologically faithful works hitherto would be Pigorass [16] and Ekeberg's cat [17]. These works combine mono and biarticular muscles in a skeletal structure, allowing the study of individual muscles during locomotion. However, Pigorass adopts only ten active muscles for its entire body, which has simplified forelimbs to reduce the total degree of freedom, not resembling feline walking. Ekeberg, on the other hand, proposed a groundbreaking cat simulation with 7 active muscles per leg, performing stable walking even with disturbances, such as weight variation, slopes and lateral forces.

In this paper we introduce Pneupard, which is a pneumatic quadruped robot with 10 active muscles on the spine and 28 active muscles along its limbs, being the most comprehensive platform so far to mimic a feline musculoskeletal structure. Drawing inspiration from a cheetah, the compliance of biological muscles is replaced by compliant air muscles, while parameters such as overall dimensions, moment arms and joint angles are faithfully reproduced.

We validate the robot design by analyzing its ability to walk on a treadmill with two hindlimbs attached to a sliding strut. Beyond performing quadruped walking, the main purpose of Pneupard is to help us understand how animals can perform adaptive locomotion, analyzing muscular contribution to the self-stability of quadruped animals and understanding the influence of such complex limbs and compliant spine on different gait patterns and their transitions.

\section{DESIGN}

The design of Pneupard takes into account, similarly to cheetah-based robots [10] [11] [14], the dimensions from its biological counterpart. Forelimbs, hindlimbs and inter-girdle measurements were considered from published data with cheetah cadavers [2] [3], marker-based observation [1] and 


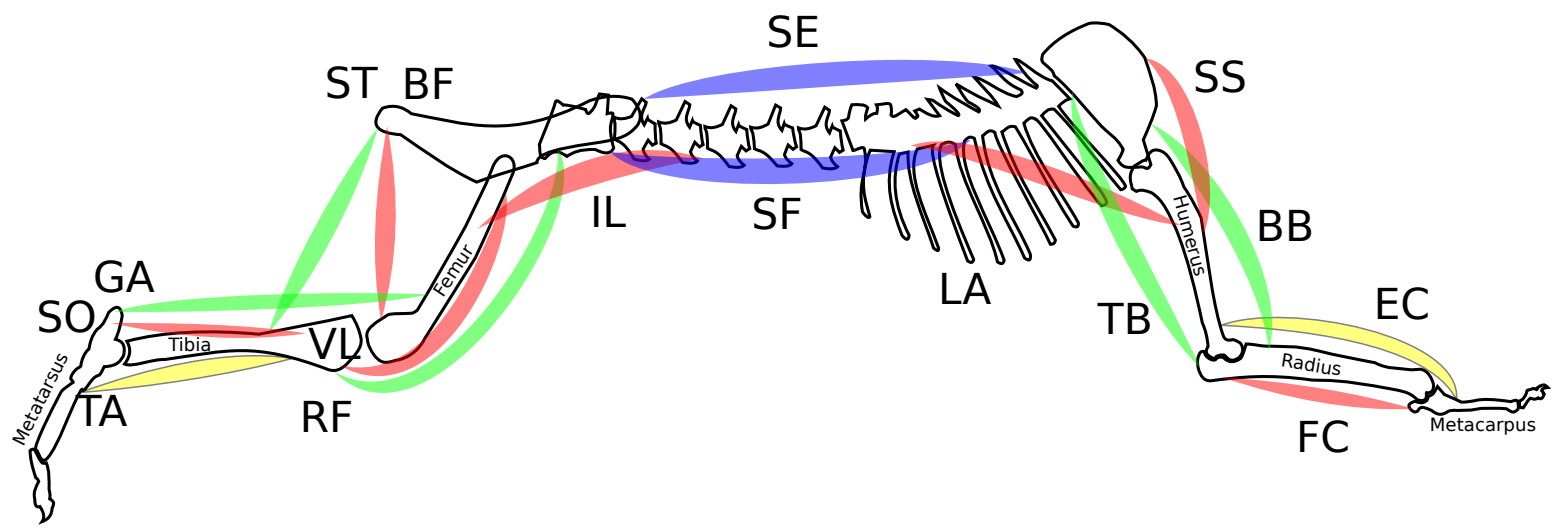

Fig. 1. Pneupard's muscle diagram. Muscles in red are monoarticular muscles, while muscles in green are biarticular. Two muscular groups are used on the spine, depicted in blue, with 5 muscles each. Muscles in yellow are passive and muscles related to adduction and abduction are not depicted on this diagram.

video observation [14], contributing to a realistic representation. However, differently from the aforementioned robots, this robot possess a highly redundant muscular structure with 46 muscles, from which 38 are actively controlled, with 4 active degrees of freedom per limb and 6 on the spine. Moreover, the number of actuators is greater than joints, simulating phenomena observed in redundant structures in biological structures.

Such muscular morphology was chosen based on biological references [5], considering muscular importance for locomotion, and the same is depicted in Fig. 1. Hind and forelimbs have four active degrees of freedom, which are adduction/abduction and flexion/extension at shoulders/hips, and flexion/extension on the other two joints (knee/elbow and ankle/wrist). The hindlimb [18] is composed of ten muscles: five monoarticulars, which are biceps femoris ${ }^{1}(\mathrm{BF}$, hip extension), iliopsoas (IL, hip flexion), vastus lateralis (VL, knee extension), soleus (SO, ankle extension) and a passive tibialis anterior (TA, ankle flexion), while also having three biarticular muscles, namely semitendinosus (ST, hip extension and knee flexion), rectus femoris (RF, hip flexion and knee extension) and gastrocnemius (GA, knee flexion and ankle extension) and two muscles responsible for adduction (passive) and abduction (active), such as gracilis, caudofemoralis or pectineus.

The forelimb is composed of eight muscles, with three monoarticulars: latissimus dorsi (LA, shoulder flexion), supraspinatus (SS, shoulder extension) and flexor carpi ulnaris (FC, wrist flexion). For biarticular roles we adopted triceps brachii (TB, shoulder flexion and elbow extension), biceps brachii (BB, shoulder extension and elbow flexion) and a passive extensor carpi radialis (EC, elbow flexion and wrist extension). Similarly to the hindlimbs, one active abductor and passive adductor, such as infraspinatus and pectoralis.

\footnotetext{
${ }^{1}$ Although biceps femoris is not a monoarticular muscle per se, the minute size of its moment arm around the knee directed us to a simplification, approximating it to zero.
}

Finally, the spine is composed of two major groups of five muscles each: one group, called spinal flexors (SF), represents abdominal muscles such as rectus abdominis, external and internal abdominal oblique and linea alba, flexing the spine, while the second group, called spinal extensors (SE) is responsible for extending the vertebral column, similarly to the sacrospinalis group (semispinalis, longissimus and iliocostalis).

\section{A. Air muscles validation as a bio-inspired alternative}

While animals possess biological muscles, which provide actuation combined with compliance to interact with the environment, the same so far is not available for human-created structures. Alternatives such as series elastic actuators [19] came into play for almost 20 years, with some robots effectively using it [20]. However, when it comes to powerto-weight ratio, actuators such as air muscles outperform any other alternative, reaching ratios as high as 400:1, being successfully used in robotic application [21].

The principle of activation is based on the intake of air through a pneumatic valve, which generates a contraction of the muscle, while exhausting the same air relaxes the muscle. The compliance offered by the actuator is inversely proportional to the muscle contraction, while the force provided by the same is, as mentioned in [22], dependent on the internal pressure and the deformation, as shown in the following equation:

$$
F \propto \frac{p_{\text {air }}}{\Delta l / L_{0}}
$$

where $F$ is the force, $p_{\text {air }}$ is the internal pressure, $L_{0}$ is the relaxed length and $\Delta l$ is the deformation after muscular activation. The resemblance between these artificial muscles and biological ones, when it comes to behavior and contraction principle, have led many researchers to adopt these air muscles for rehabilitation purposes [23]. 
TABLE I

PNEUPARD'S KEY CHARACTERISTICS

\begin{tabular}{c|c}
\hline Property & Value \\
\hline Forelimb length & $665 \mathrm{~mm}$ \\
\hline Hindlimb length & $810 \mathrm{~mm}$ \\
\hline Body height & $600 \mathrm{~mm}$ \\
\hline Inter-girdle length & $850 \mathrm{~mm}$ \\
\hline Body width & $300 \mathrm{~mm}$ \\
\hline No of degrees of freedom & 22 \\
\hline No of valves & 40 \\
\hline No of active muscles & 38 \\
\hline No of passive muscles & 8 \\
\hline Forelimb weight & $450 \mathrm{grams}$ \\
\hline Hindlimb weight & $600 \mathrm{grams}$ \\
\hline Estimated total weight & $6.4 \mathrm{~kg}$ \\
\hline
\end{tabular}

\section{B. Structure and overall dimensions}

Bones play an important role when it comes to locomotion. In [2] it is shown that the cheetah possess thicker hindlimb bones than the greyhound, leading us to believe that this extra reinforcement is needed to attain high-speed locomotion. Similarly, we approach the problem by adopting strong materials with low density, trying to keep a low inertia moment for high frequency movements.

The structure is made of carbon fiber shafts connected through ABS plastic. To lessen the stress in critical parts, such as joints, a $2 \mathrm{~mm}$ thick plate of magnesium alloy is used as a reinforcement. Considering that all three materials have density below $2 \mathrm{~kg} \mathrm{dm}^{-3}$, the robot can achieve an overall weight below $7 \mathrm{~kg}$. In Table I the specs for the robot are shown.

The compressed air needed for the air muscle operation is provided through a tether and redistributed to each of the muscles through pneumatic valves. Due to the biomimetic purpose of this robot, studies regarding energy consumption were not performed, considering that our bench tests evaluate dynamical behavior regardless of air consumption. Stronger muscles use higher flow rate 3-position pilot operated valves, while weaker ones use two small poppet valves (one for supply, one for exhaust). These valves are connected to an ARM-based microcontroller, called Adaptive Board, which communicates with a computer through serial protocol.

As a biomimetic platform, the control method should be capable of replicating a muscular activation pattern (MAP). Adopting 4 basic stages of stepping (touch down, stance, lift off and swing, as explained in [4]), where each stage has muscular air pressure proportional to EMG values observed in walking cats, we adopted rules to perform transitions between such phases: when force is applied on the foot during touch down, the stage transitions to stance; when this force decreases below a fixed threshold, lift off starts; when this force becomes zero, the leg swings; after a predetermined timer, the leg adopts touch down again.

The pressure inside each muscle can be measured by

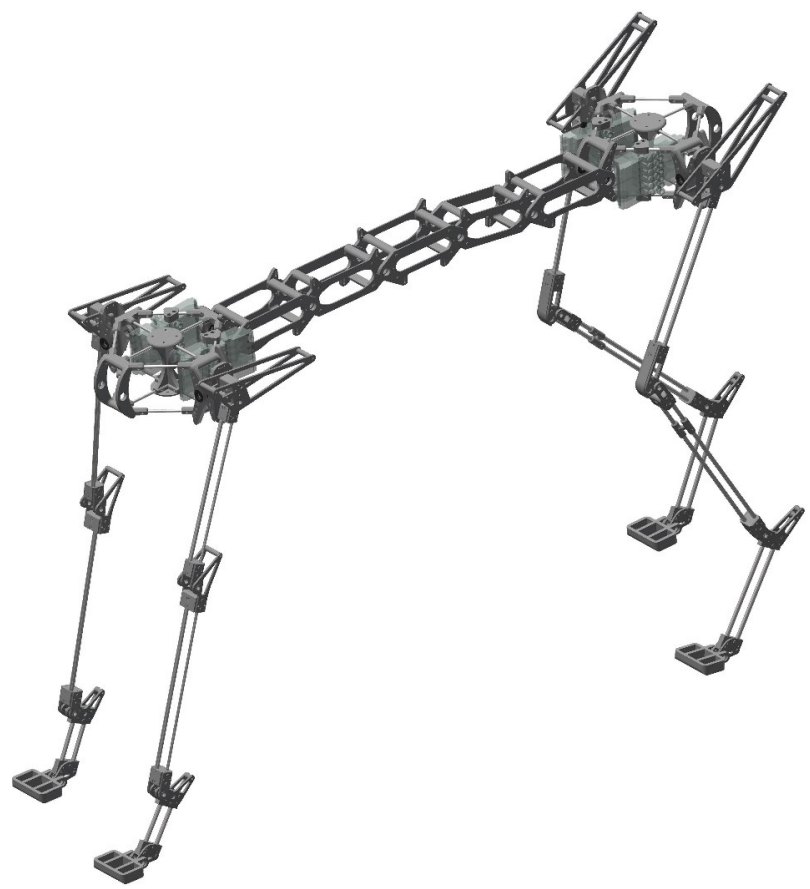

Fig. 2. Ongoing development of the full-body Pneupard. Its complex muscular structure contributes to the understanding of animals and their locomotion.

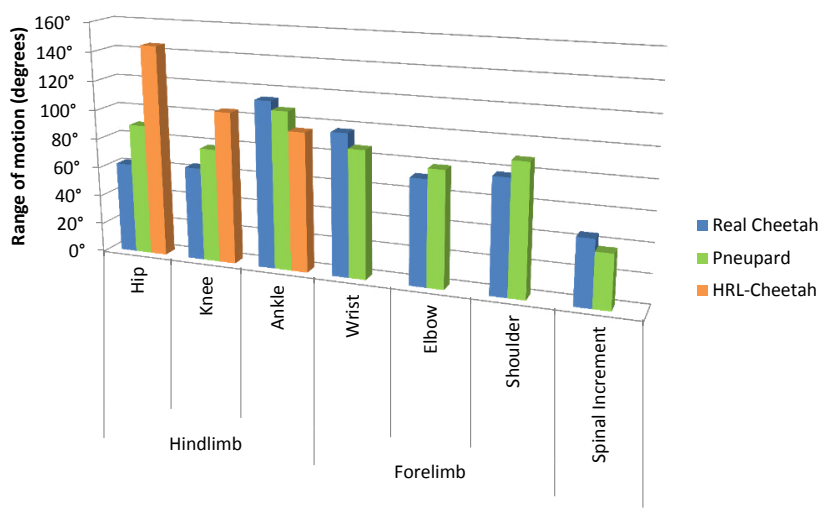

Fig. 3. Range of motion of joints. The values for HRL Cheetah are only valid for hindlimbs, and the value for its hip joint has the spinal increment added to it. Live cheetah forelimb and spinal values are based on observations, while hindlimbs are based on [2].

SMC's PSE540 sensors, allowing us to have a better control of leg placement. Although gyroscopes and accelerometers might improve stability, to better simulate decerebrate cat's behavior we opted for an almost sensorless assembly. A picture of the full robot in its design process is shown in Fig. 2.

\section{Range of motion and moment arms consideration}

Pneupard's range of motion and moment arms are based on published data from biologists [1] [2] [3] and roboticists [14]. The range of motion of a feline such as a cheetah deserves special attention due to its highly flexible spine. The values registered for hip and shoulder range of motion 


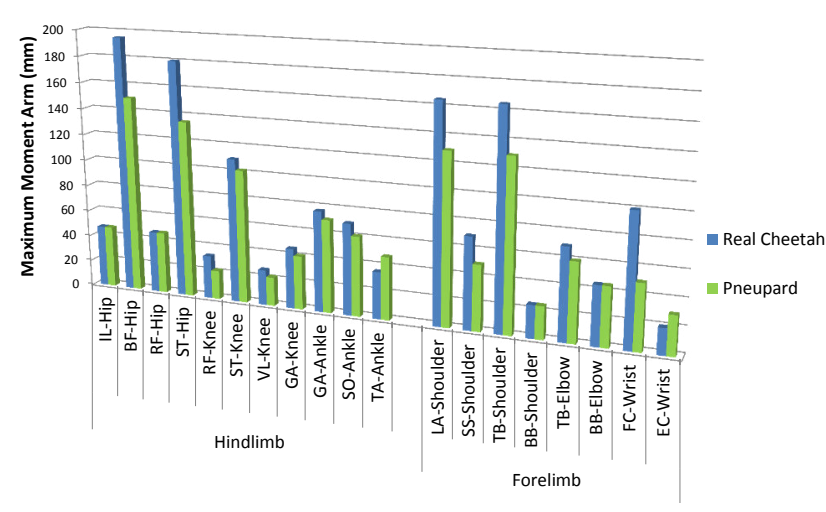

Fig. 4. Maximum moment arms comparison between Pneupard and a real cheetah. Values to the right correspond to hindlimb, while we have forelimb values to the left. Animal values based on [2] and [3].

vary drastically if the spinal contribution is considered, as pointed out by Hoffmann [14].

In Fig. 3 we show a comparative chart between forelimb, hindlimb and spine range of motion with data extracted from animal observation, compared to Pneupard and the robotic cheetah developed by HRL laboratories[14]. In the spinal increment column the contribution to hip and shoulder joints from the spine is demonstrated, and Pneupard's values range closely to the biological estimation.

Moment arm changes alter the behavior of the robot by changing the effective mechanical advantage, which acts similarly to a gearbox inside a car. While short moment arms allow fast movements with low torque, long moment arms permit very high torques associated with slow movement. More important than forelimb and hindlimb dimensions (which was proved very similar between felines by [1]), a true biomimetic approach should consider the underpinnings of the mechanical power: muscular structure.

While in [3] Wilson proved that cheetahs possess longer moment arms than greyhounds, keeping similar dimensions and weight, in Fig. 4 we show a moment arm comparison between our robot and a cheetah. Moment arms for extension movements in hips and ankles are much bigger than flexion for hindlimbs, while the opposite is true for forelimbs, leading to faster swing phases and slower stance phases, improving the duty factor. Moreover, extreme values, such as hips and shoulders moment arms, had to be decreased to fit our mechanical constraints (pneumatic muscles and weight limitations).

\section{EXPERIMENTS AND RESULTS}

Although the construction of the robot is still ongoing, we could perform preliminary experiments with a prototype, evaluating the feasibility of the project. To prove the biomimetic value of the robot we adopted as control method a MAP with four different stages: stance, lift off, swing and touch down. In Fig. 5 we show the adopted activation pattern as a function of pressure for all 7 muscles used for flexion/extension movements. This pattern is based on data from EMG signals extracted from cats walking

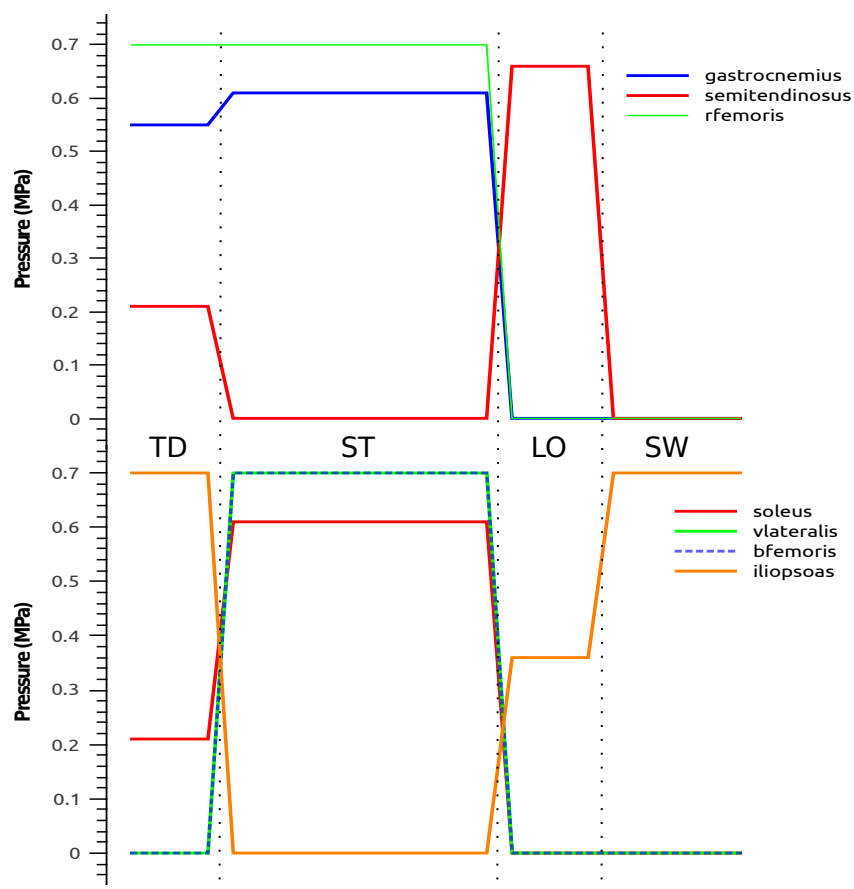

Fig. 5. Activation pattern for the hindlimbs. On the upper inset we have biarticular muscles, while on the lower one monoarticular muscles are depicted. The four phases of the gait (TD, ST, LO and SW) are defined as touch down, stance, lift off and swing, respectively.

on a treadmill, and adjusted to compensate for differences between artificial and biological muscles.

The suggested pattern is used on a bipedal arrangement of the robot, obtained by attaching the hindlimbs to a sliding strut, similarly to simulations performed in [17]. This assembly was put to test on a treadmill, running at a constant speed of $0.8 \mathrm{~km} \mathrm{~h}^{-1}$, in order to verify the walking stability of the hindlimbs with the suggested pattern. As a ground force feedback (to switch phases) a single FSR sensor was installed on each hindlimb.

Differently from Ekeberg, this robot not only brings to a real environment the stepping feline, but also considers influences of different muscular pressures on the gait stability. Moreover, the use of feline-inspired moment arms may give better insights on self-stability than a simplified strategy adopted on [17] and described in its appendix. Angular position sensors were not used on the hips to limit swing movement, but a stable walking was still possible without them.

While the robot walks on the treadmill, motion capture systems are used to acquire data pertaining to the robot's position and angles. In Fig. 6 the angles related to the robot's hip, knee and ankle are shown. The high level of stability obtained by this simple MAP, combined with ground reaction forces feedback leaded us to a more rigorous test: introducing $26 \mathrm{~mm}$ disturbances along the walking path of the treadmill and registering how it affects the gait stability.

In Fig. 7 we have a 60 seconds walk on the treadmill with 5 different obstacles randomly placed. As a stability measuring criteria we registered the influence of these disturbances 

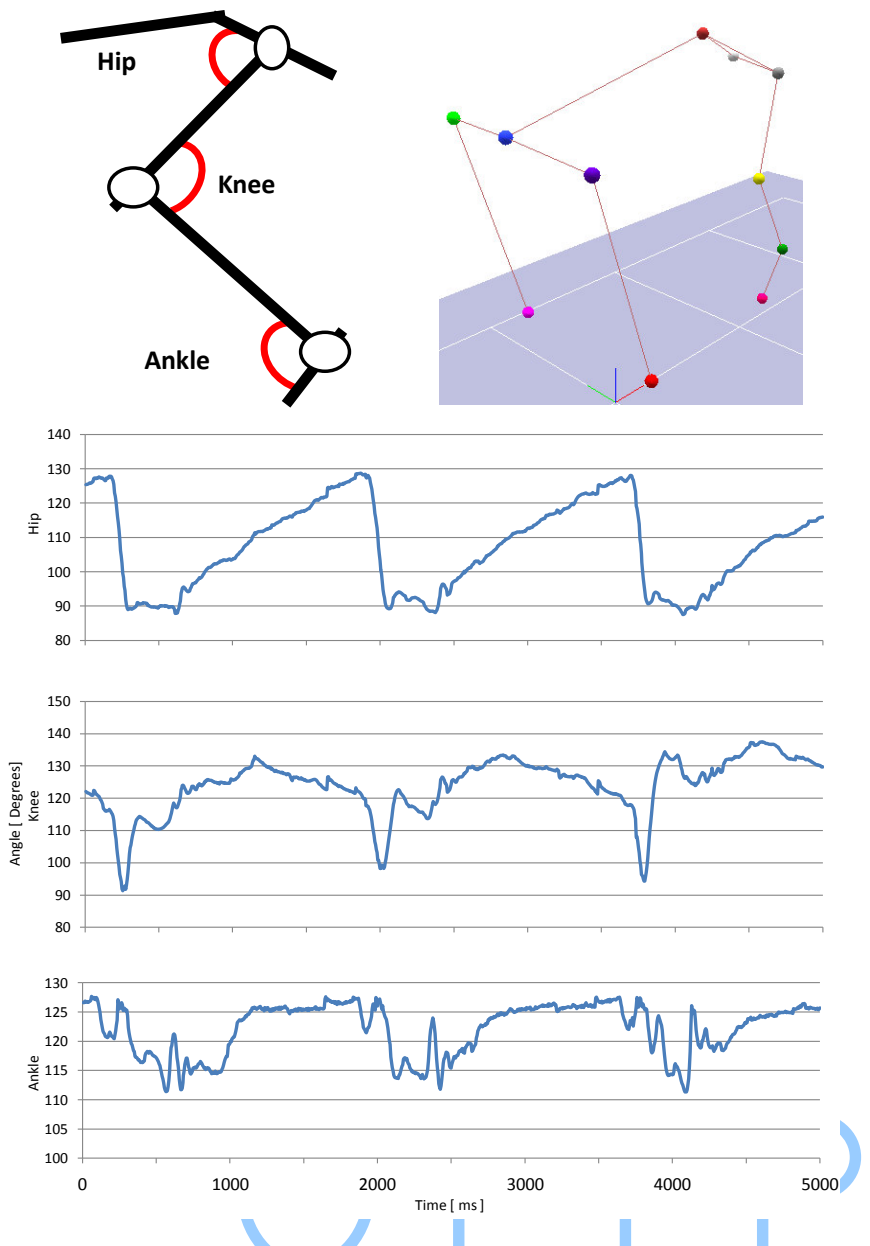

Fig. 6. Hindlimb angles during walking on a treadmill, extracted with a motion capture system. From top to bottom, we have a pictogram of the assembly (markers only on left hindlimb), hip, knee and ankle angles.

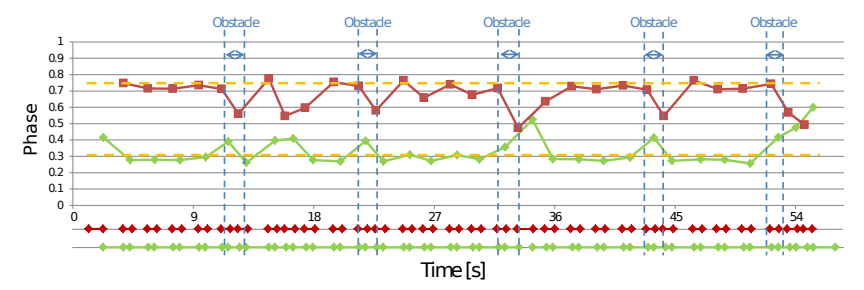

Fig. 7. Phase difference during walking on a treadmill with five $26 \mathrm{~mm}$ obstacles randomly placed. The right hindlimb is depicted in green, while the left is depicted in red.

on the phase difference between right and left hindlimbs. Similarly to [17], the phase difference is defined by:

$$
\Phi_{H(\text { actual })}=\frac{T_{H(\text { actual })}-T_{O(\text { previous })}}{T_{O(\text { next })}-T_{O(\text { previous })}}
$$

where $\Phi_{H}$ is the specified hindlimb's phase, $H$ and $O$ standing for hindlimb and opposite hindlimb and $T$ standing for the moment in which the hindlimb touches the floor. The idea is that the phase of each hindlimb is defined in association with the floor touching moments of the opposite hindlimb.

\section{DISCUSSION}

Initially analyzing the proposed MAP (Fig. 5), we can conclude that the majority of the muscles act during stance phase, with only two exceptions: iliopsoas, which is antagonistic to biceps femoris, thus relaxing when the latter is at work, and semitendinosus.

Biological observations [5] have implied that the role of semitendinosus is mainly flexor, while in computer simulations [17] Ekeberg suggested that the role of semitendinosus is related with lift off and touch down phases. The biarticular characteristic of the muscle is hypothesized to aid during phase changes.

The remaining 5 muscles ( 2 biarticulars, 3 monoarticulars) are used to lift the hindquarters and thrust the whole system forward. While monoarticular muscles generate force locally, it is known that biarticulars contribute to force transfering between joints [15].

Although MAP help us understand better the inner workings of animals, a great shortcoming on using highly compliant air muscles would be the degrading on controllability when compared with traditional actuators. Although the controllability might not be satisfactory for precise industrial applications, we believe that the current precision, combined with great lightness, might prove ideal for everyday robotic application.

In Fig. 6 the hip angles are shown, and a parallel between these and the MAP can be traced. As explained above, iliopsoas is the main responsible for hip flexion, being thus responsible for the quick change on the hip angle (quick downward change from $128^{\circ}$ to $\left.90^{\circ}\right)$. At the ankle joint, the elasticity of tibialis anterior (passive muscle in our setting) promotes dorsiflexion when the extensors soleus and gastrocnemius are relaxed. During stance this joint has a very low angle variability, agreeing with similar observation on biological cats [5].

On the knee joint, even though this robot does not possess any muscle specifically for knee flexion (similarly to [17]), knee flexion is obtained through ballistic movement (similarly to passive walkers), being enough to produce swing clearance and even overcome obstacles.

In Fig. 7 we have the hindquarter and hindlimbs walking 62 steps on a treadmill. While the approximate undisturbed phase for the right limb is 0.3 , the left one is approximately 0.7, which differs from Ekeberg's work, where both limbs had a phase of 0.5 when walking stably. We are led to believe that while a computer simulation can produce perfectly symmetric muscles, small length differences associated with the air muscles construction method accounted for an uneven gait. Nonetheless, the walking stability was great enough to overcome these and other disturbance sources.

The introduction of random disturbances ${ }^{2}$ affects the phase stability, shortening swing/touch down durations on one leg, which accelerates lift off on the opposite one. After the disturbance, the system returns to the original state attractor with stable phases.

\footnotetext{
${ }^{2}$ Video on treadmill, keyword "Pneupard" on www.youtube.com.
} 


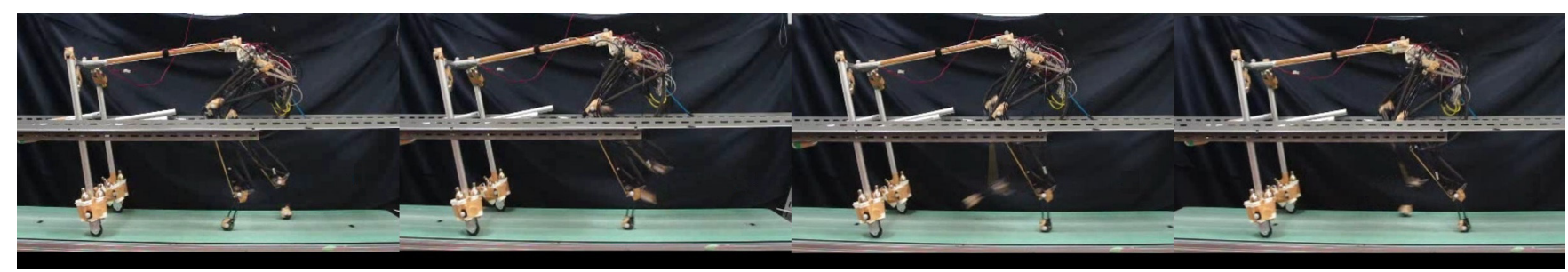

Fig. 8. Snapshots of Pneupard walking on the treadmill. While the left hindlimb stays in stance phase, the right hindlimb starts in lift off, pass through swing and ends in a touch down phase.

Finally in Fig. 8 we can see the experimental assembly walking on the treadmill. Guides were installed on both sides of the treadmill to prevent the robot from falling sideways and rubber pads were used on the tip of the foot to compensate the low friction from the treadmill.

During experiments Pneupard reproduced feline stepping while withstanding disturbances, similarly to Ekeberg. However, the introduction of real life noise, construction related errors and the lack of an angular position sensor generated differences between simulation and experiment. Beyond Ekeberg's work, currently we are exploring muscular synergy contribution to gait stability, as well as individual muscle contribution to self-stability.

Cats from various sizes are notorious for great skills, such as jumping, running or climbing, and even though they possess great similarities in muscle and bone number with human beings, we are not as agile as they are. Certain that a better performance is not associated with a more sophisticated brain capacity (the brain of a domestic cat weighs only 30 grams), we tackled the problem by reconstructing the animal morphology to understand it.

In this paper we studied the animal morphology and recreated it with a degree of details never seen before. We conducted experiments and produced a stable walking, withstanding disturbances on a treadmill.

In the future we plan on connecting the spine and forelimbs (under construction) to the robot and reproducing quadrupedal walking while exploring muscular contributions to stability. As a long term objective we believe that it will be possible to develop better robots, based on self-stability knowledge gained from animals.

\section{REFERENCES}

[1] L. Day and B. Jayne, Interspecific scaling of the morphology and posture of the limbs during the locomotion of cats (Felidae), J. Exp. Biol., vol. 210, 2007, pp. 642-657.

[2] P.E. Hudson, A.M. Wilson et al., Functional anatomy of the cheetah (Acinonyx Jubatus) hindlimb, J. Anat., vol. 218, 2011, pp. 363-374.

[3] P.E. Hudson, A.M. Wilson et al., Functional anatomy of the cheetah (Acinonyx Jubatus) forelimb, J. Anat., vol. 218, 2011, pp. 375-385.

[4] I. Engberg and A. Lundberg, An electromyographic analysis of muscular activity in the hindlimb of the cat during unrestrained locomotion, Acta Physiol. Scand., vol. 75, 1969, pp. 614-630.

[5] G.E. Goslow Jr., R.M. Reinking, and D.G. Stuart, The cat step cycle: hind limb joint angles and muscle lengths during unrestrained locomotion, J. Morphol., vol. 141, 1973, pp. 1-42.

[6] A. English, An electromyographic analysis of forelimb muscles during overground stepping in the cat, J. Exp. Biol., vol. 76, 1978, pp. 105122.
[7] B.I. Prilutsky, W. Herzog, and T.L. Allinger, Mechanical power and work of cat soleus, gastrocnemius and plantaris muscle during locomotion: possible functional significance of muscle design and force patterns, J. Exp. Biol., vol. 199, 1996, pp. 801-814.

[8] N.G. Tsagarakis, B. Vanderborght, Y. Yousheng, and D.G. Caldwell, HyQ - Hydraulically actuated quadruped robot: Hopping leg prototype, in Proc. Int. Conf. Biomed. Robot. and Biomechatronics, 2008, pp. 593-599.

[9] Y. Fukuoka, H. Kimura, and A.H. Cohen, Adaptive dynamic walking of a quadruped robot on irregular terrain based on biological concepts, J. Robot. Res., vol.22, 2003, pp.187-202.

[10] A. Sproewitz, A.J. Ijspeert et al., Oncilla robot : a light-weight bioinspired quadruped robot for fast locomotion in rough terrain, in Proc. Fifth Int. Symp. Adaptive Motion on Animals and Machines, Osaka, Japan, 2011.

[11] A.K. Valenzuela and K. Sangbae, Optimally scaled hip-force planning: A control approach for quadrupedal running, in Proc. Int. Conf. on Robotics and Automation, 2012, pp. 1901-1907.

[12] K. Aschenbeck, N. Kern, R. Bachmann, and R. Quinn, Design of a quadruped robot driven by air muscles, in Proc. Int. Conf. Biomed. Robot. and Biomechatronics, 2006, pp. 875-880.

[13] K. Tsujita, T. Kobayashi, T. Inoura, and T. Masuda, Gait transition by tuning muscle tones using pneumatic actuators in quadruped locomotion, in Proc. Int. Conf. on Intelligent Robots and Systems, 2008, pp. 2453-2458.

[14] M. Lewisy, M. Buntingy, B. Salemi, and H. Hoffmann, Toward ultra high speed locomotors: design and test of a cheetah robot hind limb, in Proc. Int. Conf. on Robotics and Automation, 2011, pp. 1990-1996.

[15] G.J. van Ingen Schenau, M.F. Bobbert, and R.H. Rozendal, The unique action of bi-articular muscles in complex movements, J. Anat., vol. 155, 1987, pp. $1-5$.

[16] Y. Yamada, S. Nishikawa, Y. Kuniyoshi et al., Neural-body coupling for emergent locomotion: a musculoskeletal quadruped robot with spinobulbar model, in Proc. Int. Conf. on Intelligent Robots and Systems, 2011, pp. 1499-1506.

[17] O. Ekeberg and K. Pearson, Computer simulation of stepping in the hind legs of the cat: an examination of mechanisms regulating the stance-to-swing transition, J. Neurophysiol., vol. 94, 2005 , pp. 42564268.

[18] A. Rosendo, K. Narioka, and K. Hosoda, Muscle roles on directional change during hopping of a biomimetic feline hindlimb, in Proc. Int. Conf. on Robotics and Biomimetics, 2012.

[19] G.A. Pratt and M.M. Williamson, Series elastic actuators, in Proc. Int. Conf. on Intelligent Robots and Systems, 1995, pp. 399-406.

[20] M. Hutter et al., Starleth: a compliant quadrupedal robot for fast, efficient and versatile locomotion, in Proc. Int. Conf. on Climbing and Walking Robots, 2012.

[21] K. Hosoda, Y. Sakaguchi, H. Takayama, and T. Takuma, Pneumaticdriven jumping robot with anthropomorphic muscular skeleton structure, Auton. Robot., vol. 28, 2010, pp. 307-316.

[22] I. Boblan, A human-like robot hand and arm with fluidic muscles: biologically inspired construction and functionality, Embodied Artificial Intelligence, Springer, Dagstuhl Castle, 2004.

[23] S.Q. Xie and P.K. Jamwal, An iterative fuzzy controller for pneumatic muscle driven rehabilitation robot, Expert Systems with Applications, vol. 38, 2011, pp. 8128-8137.

[24] A.J. Ijspeert, Central pattern generators for locomotion control in animals and robots: A review, Neural Networks, vol. 21, 2008, pp. 642-653. 